\title{
Crystal structure of parallel-stacked peryleneimides and their application to organic field-effect transistor devices
}

\author{
Kazuyuki Sato ${ }^{\text {a) }}$ and Jin Mizuguchi \\ Department of Applied Physics, Graduate School of Engineering, Yokohama National University, \\ 240-8501 Yokohama, Japan \\ Yoshimasa Sakai and Shinji Aramaki \\ Mitsubishi Chemical Group Science and Technology Research Center, Inc., 227-8502 Yokohama, Japan
}

(Received 7 August 2007; accepted 29 October 2007; published online 10 January 2008)

\begin{abstract}
Organic field-effect transistors (FETs) have recently attracted attention mainly because of the low fabrication cost. Parallel-stacked structure is considered favorable for realizing a high mobility along the stacked direction in organic FETs. Among our dipyridyl-peryleneimides synthesized previously for $\mathrm{H}_{2}$ sensors (OPP, MPP, and PPP for $o_{-}^{-}, m$-, and $p$-position of the $\mathrm{N}$ atom in the pyridyl ring, respectively), OPP and PPP are found to crystallize in this structure and are expected to show better FET characteristics than MPP. Therefore, the FET characteristics have been studied in the present investigation from the standpoint of the crystal structure. The FET devices based on OPP and PPP exhibit $n$-type characteristics, showing a mobility of about $10^{-5} \mathrm{~cm}^{2} /(\mathrm{V} \mathrm{s})$ and "on/off" ratios of about $10^{2}$, whereas only poor performance was observed for the MPP-based FET. (ㄷ) 2008 American Institute of Physics. [DOI: 10.1063/1.2827483]
\end{abstract}

\section{INTRODUCTION}

Organic field effect transistors (FETs) have attracted much attention in recent years because of their several potential applications such as large-area coverage, structural flexibility, and especially low cost electronic devices. ${ }^{1}$ They have been under widespread development for active-matrix flat-panel displays, sensors, and radio-frequency identification tags (RFIDs), making use of their advantages. The fieldeffect mobilities $\mu$ of the FETs with organic molecules are still much lower than those of the conventional FETs based on inorganic materials. Because of this, new organic materials are being extensively studied in an attempt to achieve FET devices with high $\mu$ value. ${ }^{2}$

A recent publication on pentacene ${ }^{3}$ has pointed out that a conductivity path is achieved along the stacking axis due to the orbital overlap. Because of this, the parallel-stack structure is considered ideal for realizing a high mobility in organic semiconductors. However, the complete parallel stack (i.e., complete "atom/atom" contact) seems to be rather improbable according to our understanding because of the "out of phase" repulsive interaction between perfectly parallelstacked molecules, as typically shown by the structure of paracyclophanes. ${ }^{4}$ For example, [2.2] paracyclophane has two benzene rings which are rigidly held together by the ethano bridges in para-positions, as shown in Fig. 1(a). X-ray structure analysis revealed that the two benzene rings are heavily deformed due to repulsive out of phase interactions between atomic orbitals [Fig. 1(b)]. For this reason, the molecules arrange themselves to minimize the repulsive interaction along the stacking axis, in such a way as to slide in a fashion "herringbone" [Fig. 2(a)] or to rotate in manner "quasiparallel-stack" [Fig. 2(b)]. The herringbone structure is quite

${ }^{a)}$ Electronic mail: d07sd201@ynu.ac.jp. common in most organic semiconductors, whereas the quasiparallel-stack is rather rare, but is expected to provide us with a high mobility.

We have previously synthesized novel dipyridylperyleneimide derivatives for $\mathrm{H}_{2}$ gas sensors analyzed their crystal structure. ${ }^{5}$ The pyridyl ring in which the $\mathrm{N}$ atom is positioned at the ortho-, meta-, or para-site is directly connected to the peryleneimide skeleton, as shown in Fig. 3. These are called OPP, MPP, and PPP, respectively. Among these, OPP and PPP are found to crystallize in quasi-parallelstack structure. Therefore, we believed that OPP and PPP could basically exhibit better FET performance than MPP.

The objective of the present investigation is to study the FET characteristics of OPP, MPP, and PPP from the standpoint of the crystal structure.

\section{SUMMARY OF THE STRUCTURE ANALYSIS}

Table I details the crystallographic parameters for OPP, ${ }^{6}$ MPP, ${ }^{7}$ and PPP. ${ }^{8}$ In the unit cell of OPP, there are two independent molecules, $\mathrm{A}$ and $\mathrm{B}$. Both molecules $\mathrm{A}$ and $\mathrm{B}$ are characterized by $C_{i}$ symmetry. Two pyridyl rings are twisted in the same direction with respect to the peryleneimide skel- (a)

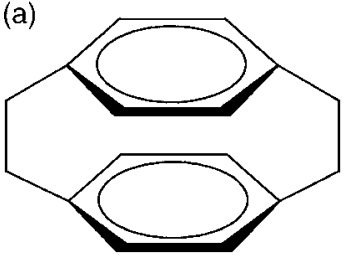

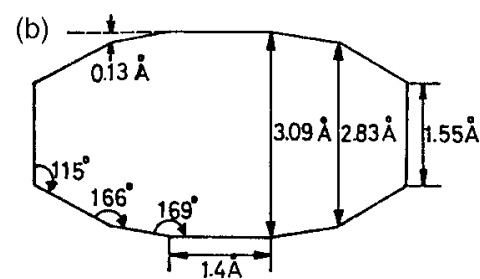

FIG. 1. [2.2]paracyclophane: (a) molecular conformation and (b) side view of the structure. 

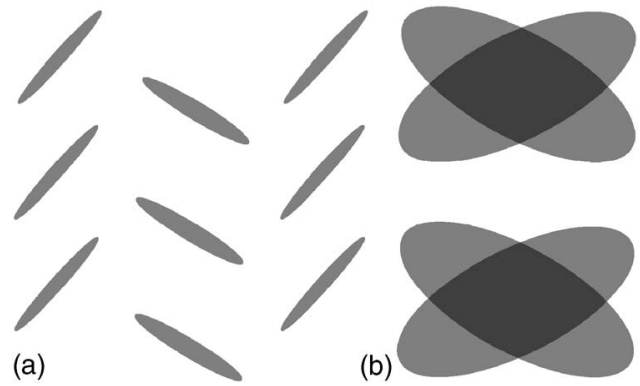

FIG. 2. (a) "Herringbone" structure (side view) and (b) quasi-parallel-stack structure (top view).

eton by $77.7^{\circ}$ in molecule $\mathrm{A}$ and $72.8^{\circ}$ in molecule B. Molecules $\mathrm{A}$ and $\mathrm{B}$ are stacked alternately with a tilt angle of about $39^{\circ}$ shown in Fig. 4(a).

The molecule of MPP is characterized by $C_{i}$ symmetry. Two pyridyl rings are twisted in the same direction by $54.9^{\circ}$. The molecules are stacked in a fashion "hunter's fence" (viz., when viewed from the side, the molecules, slipped by $45^{\circ}$ within molecular stacks, cross each other in a fencelike structure) along the $b$ axis, as shown in Fig. 4(b).

Contrary to OPP and MPP molecules, the PPP molecule has a $C_{2}$ symmetry. Therefore, two pyridyl rings are twisted in opposite directions by $74.5^{\circ}$. The molecules are stacked along the $c$ axis with a tilt angle of $32^{\circ}$, as shown in Fig. 4(c), and the molecular plane is exactly perpendicular to the stacking axis, showing a typical structure of quasi-parallelstack.

\section{EXPERIMENT}

\section{A. Synthesis and purification of the material}

OPP was synthesized by reaction of perylenetetracarboxylic dianhydride with 2-aminopyridine at $490 \mathrm{~K}$ in dimethylnaphthalene for $3 \mathrm{~h} .{ }^{9}$ Likewise, MPP and PPP were synthesized with 3-aminopyridine and 4-aminopyridine, respectively. The products were purified three times by sublimation at $750 \mathrm{~K}$ using a two-zone furnace. ${ }^{10}$

\section{B. Fabrication of the FET}

The FET structure is depicted, as shown in Fig. 5. A $\mathrm{SiO}_{2}$ layer of about $300 \mathrm{~nm}$ was thermally grown on heavily doped $n$-type Si substrate which serves as the gate electrode. On top of $\mathrm{SiO}_{2}$, the source and drain electrodes made of $\mathrm{Au} / \mathrm{Cr}$ were prepared by photolithographic technique. The channel length and the channel width were 10 and $500 \mu \mathrm{m}$,

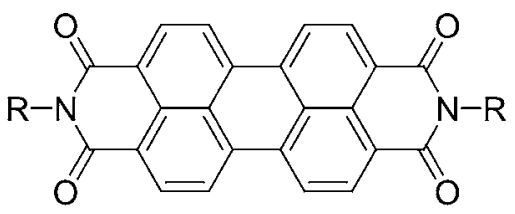

(a)

(b)

(c)

$R$ :

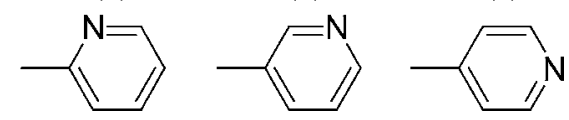

FIG. 3. Molecular structure of peryleneimide derivatives: (a) OPP, (b) MPP, and (c) PPP.
TABLE I. Crystallographic parameters for OPP, MPP, and PPP.

\begin{tabular}{lccc}
\hline \hline & $\mathrm{OPP}($ ortho $)$ & MPP (meta) & $\mathrm{PPP}($ para $)$ \\
\hline Formula & $\mathrm{C}_{34} \mathrm{H}_{16} \mathrm{~N}_{4} \mathrm{O}_{4}$ & $\mathrm{C}_{34} \mathrm{H}_{16} \mathrm{~N}_{4} \mathrm{O}_{4}$ & $\mathrm{C}_{34} \mathrm{H}_{16} \mathrm{~N}_{4} \mathrm{O}_{4}$ \\
Crystal system & Monoclinic & Monoclinic & Orthorhombic \\
Space group & $P 2_{1} / c$ & $P 2{ }_{1} / n$ & $P c c n$ \\
Molecular symmetry & $C_{i}$ & $C_{i}$ & $C_{2}$ \\
$Z$ & 4 & 2 & 4 \\
$a(\AA)$ & $17.599(1)$ & $15.422(2)$ & $21.232(2)$ \\
$b(\AA)$ & $7.1705(5)$ & $3.8275(6)$ & $15.890(2)$ \\
$c(\AA)$ & $20.679(2)$ & $19.282(3)$ & $6.9311(8)$ \\
$\beta(\mathrm{deg})$ & $111.004(5)$ & $103.29(1)$ & $\ldots$ \\
$d\left(\mathrm{~g} / \mathrm{cm}^{3}\right)$ & 1.49 & 1.63 & 1.55 \\
$R_{1}$ & 0.062 & 0.039 & 0.074 \\
\hline \hline
\end{tabular}

respectively. After that, each thin layer of peryleneimides was applied by vacuum evaporation to the thickness of about $70 \mathrm{~nm}$ at room temperature (Tokyo Vacuum Co. Ltd., model EG240).

\section{Equipment}

The $I-V$ characteristics of the FETs were measured at room temperature under vacuum with an Agilent $4155 \mathrm{C}$
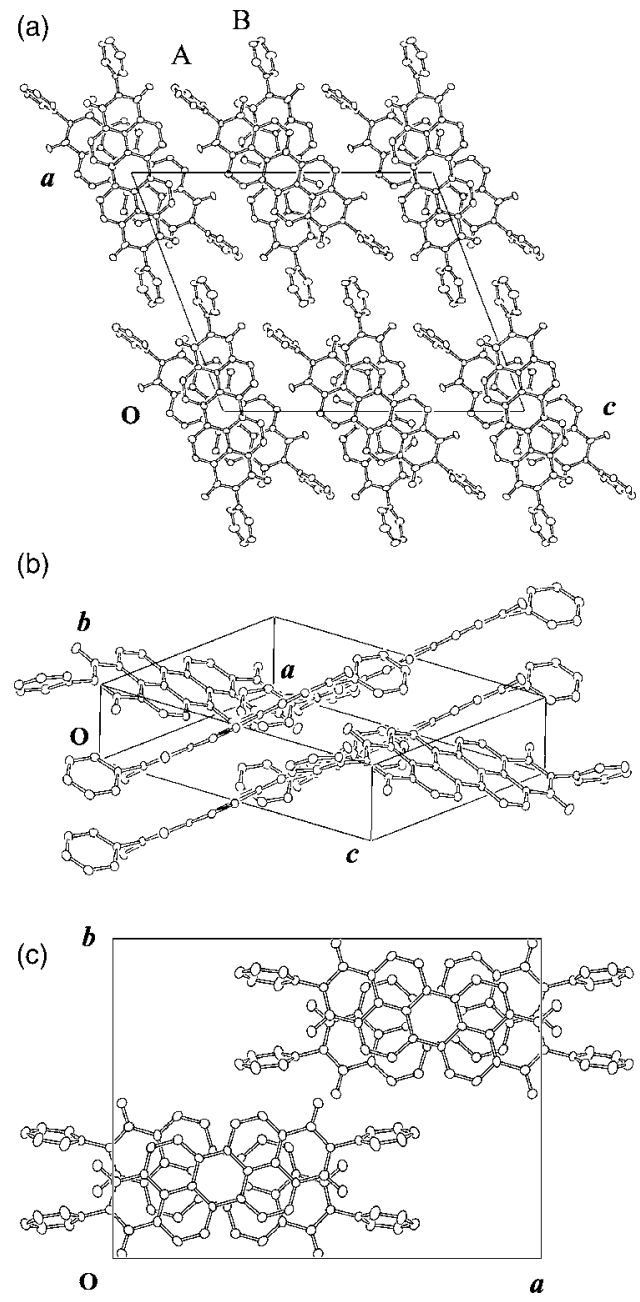

FIG. 4. Molecular arrangement: (a) projection of OPP on to the (010) plane, (b) the packing arrangement of MPP, and (c) projection of PPP onto the (001) plane. 


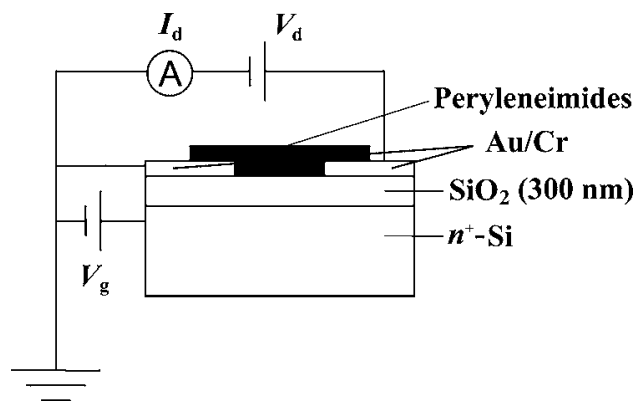

FIG. 5. Device structure of organic FET.

semiconductor parameter analyzer. X-ray diffraction diagrams of thin films were measured on Rigaku RINT2000 using graphite-monochrometed $\mathrm{Cu} K \alpha$ radiation.

\section{RESULTS AND DISCUSSION}

\section{A. FET characteristics}

Figure 6(a) shows the "drain current"-“drain voltage" $\left(I_{d}-V_{d}\right)$ plot based on OPP, which is typical of the FET char-

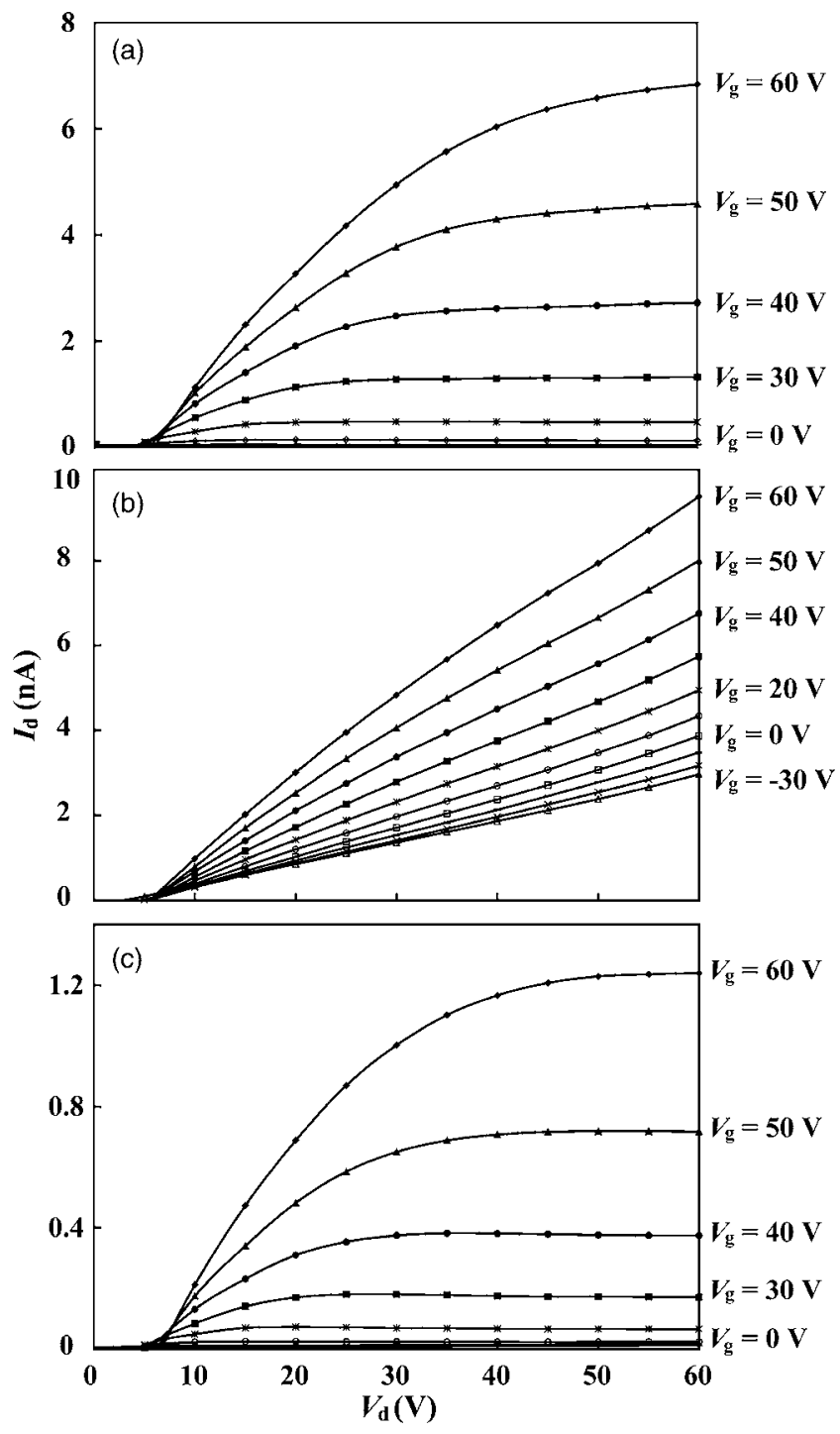

FIG. 6. I- $V$ characteristics of FETs: (a) OPP, (b) MPP, and (c) PPP.

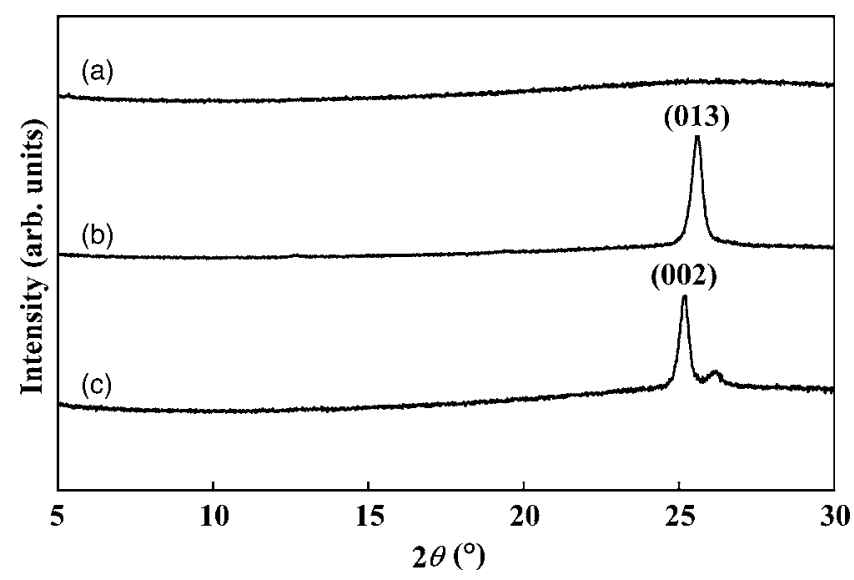

FIG. 7. X-ray diffraction diagrams on evaporated films: (a) OPP, (b) MPP, and (c) PPP.

acteristics composed of a linear region at low $V_{d}$ and a saturation region at a high $V_{d}$. The positive sign of both the drain and gate voltage $\left(V_{d}\right.$ and $\left.V_{g}\right)$ indicates an electron transport. The mobility $(\mu)$ calculated from the slope of the $I_{\text {sat }}^{1 / 2}-V_{g}$ plot in the saturated region is $8.1 \times 10^{-6} \mathrm{~cm}^{2} /(\mathrm{V} \mathrm{s})$, and the threshold voltage $V_{t}$ of the devices is $4.2 \mathrm{~V}$. Furthermore, the "on/off" ratio is 150 in OPP that is defined as the ratio of the drain current at $V_{g}=50 \mathrm{~V}$ to that at $V_{g}=-30 \mathrm{~V}$, while the drain voltage is kept constant at $V_{d}=30 \mathrm{~V}$.

Figure 6(b) shows the $I_{d}-V_{d}$ plot of the FET based on MPP. The drain current increases nearly linearly and no saturation is recognized at higher drain voltages. Consequently, the on/off ratio is quite small. This result suggests that the interface between the electrode and MPP makes an Ohmic contact, indicating that the inversion layer is not formed under the applied gate voltage.

Figure 6(c) shows the $I_{d^{-}} V_{d}$ plot of the FET based on PPP. The present FET characteristic is quite similar to those of OPP. The FET is of $n$ type and the mobility, $V_{t}$, and on/off ratio are $2.2 \times 10^{-6} \mathrm{~cm}^{2} /(\mathrm{V} \mathrm{s}), 4.6 \mathrm{~V}$, and 46 , respectively.

The above results show clearly that the FET devices based on OPP and PPP are by far superior to that of MPP. In other words, the quasi-parallel-stack structure is effective in improving the mobility value.

\section{B. Correlation between the FET characteristics and the molecular orientation on substrate}

As shown in Fig. 5, the drain current flows parallel to the substrate. Then, an important question arises whether the molecules are so arranged on substrate that quasi-parallelstack is formed in parallel to the substrate. To check this, $\mathrm{x}$-ray diffraction diagrams of evaporated films have been measured. Figures 7(a)-7(c) show the x-ray diffraction diagrams of evaporated OPP, MPP, and PPP, respectively: film thickness of about $3000 \AA$. No diffraction peak is observed in OPP which is quite indicative of an amorphous phase. On the other hand, one diffraction peak appears around $2 \theta$ $=25^{\circ}-26^{\circ}$ in MPP and PPP which is assigned to the (013) and (002) planes, respectively. These correspond to the diffraction along the stacking axis, indicating that the molecules are arranged parallel to the substrate. In other words, the 
quasi-parallel-stack structure is formed perpendicular to the substrate. These results indicate that no correlation is found between the FET characteristics and the molecular arrangement on substrate.

\section{SUMMARY AND CONCLUDING REMARKS}

Organic FETs have been fabricated on the basis of three kinds of peryleneimide derivatives: OPP, MPP, and PPP. OPP and PPP, which possess quasi-parallel-stack structure, exhibit $n$-type characteristics, showing a mobility of about $10^{-5} \mathrm{~cm}^{2} /(\mathrm{V} \mathrm{s})$ and on/off ratio of about 100. OPP and PPP with quasi-parallel-stack structure are found to show significantly better FET characteristics than MPP with absence of the parallel-stacked structure. However, no correlation is recognized between the FET characteristics and the molecular orientation on substarate.

The mobility achieved by OPP and PPP with the quasiparallel-stack structure is not at all high sufficient for practical devices. However, we believe that our experiment serves as a model to study the stack structure and provides us with information about the merit of parallel-stacked compounds. In addition, we have also pointed out that complete parallel stack structure (i.e., complete "atom/atom" contact) is improbable because of the "out of phase" repulsive interaction between the stacked molecules, as typically shown by the structure of paracyclophanes.

${ }^{1}$ C. D. Dimitrakopoulos and P. R. L. Malenfant, Adv. Mater. (Weinheim, Ger.) 14, 99 (2002).

${ }^{2}$ H. Klauk, M. Halik, U. Zschieschang, G. Schmid, W. Radik, and W. Weber, J. Appl. Phys. 92, 5259 (2002)

${ }^{3}$ J. H. Schön, Ch. Kloc, and B. Batlogg, Phys. Rev. B 63, 245201 (2001).

${ }^{4}$ A. Ishitani and S. Nagakura, Mol. Phys. 12, 1 (1967).

${ }^{5}$ K. Sato, K. Hino, H. Takahashi, and J. Mizuguchi, Int. J. Intell. Syst. 3, 52 (2007).

${ }^{6}$ J. Mizuguchi, K. Hino, K. Sato, H. Takahashi, and S. Suzuki, Acta Crystallogr., Sect. E: Struct. Rep. Online E61, 437 (2005).

${ }^{7}$ J. Mizuguchi, K. Hino, K. Sato, H. Takahashi, and S. Suzuki, Acta Crystallogr., Sect. E: Struct. Rep. Online E61, 434 (2005).

${ }^{8}$ K. Hino, K. Sato, H. Takahashi, S. Suzuki, and J. Mizuguchi, Acta Crystallogr., Sect. E: Struct. Rep. Online E61, 440 (2005).

${ }^{9} \mathrm{M}$. Herbst and K. Hunger, Industrial Organic Pigments, 3rd ed. ( $\mathrm{VCH}$, Weinheim, 2004)

${ }^{10}$ J. Mizuguchi, J. Appl. Phys. 84, 4479 (1998). 